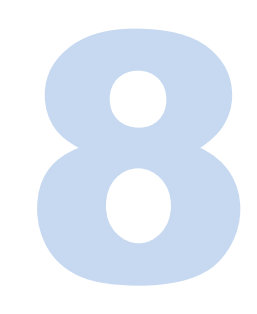

\title{
Produção do material de referência de oxitetraciclina em leite em pó desnatado
}

\section{Mychelle Alves Monteiro}

Mestre do Programa de Tecnologia de Processos Químicos e Bioquímicos da Escola de Química da UFRJ. Graduada em Química Industrial pela Universidade Federal Fluminense. Atualmente trabalha no Instituto Nacional de Controle de Qualidade em Saúde (INCQS) da Fundação Oswaldo Cruz. mychelle.monteiro@incqs.fiocruz.br

\section{Bernardete Ferraz Spisso}

Doutora do Programa de Vigilância Sanitária do Instituto Nacional de Controle de Qualidade em Saúde (INCQS) da Fundação Oswaldo Cruz. Graduada em Engenharia Química pela Universidade do Estado do Rio de Janeiro. Atualmente trabalha no Instituto Nacional de Controle de Qualidade em Saúde (INCQS) da Fundação Oswaldo Cruz.

\section{Mararlene Ulberg Pereira}

Mestre do Programa de Vigilância Sanitária do Instituto Nacional de Controle de Qualidade em Saúde (INCQS) da Fundação Oswaldo Cruz. Graduada em Licenciatura em Química pela Universidade Federal do Rio de Janeiro. Atualmente trabalha no Instituto Nacional de Controle de Qualidade em Saúde (INCQS) da Fundação Oswaldo Cruz.

\section{Rosana Gomes Ferreira}

Mestre do Programa de Tecnologia de Processos Químicos e Bioquímicos da Escola de Química da UFRJ. Graduada em Química Industrial pela Universidade Federal Rural do Rio de Janeiro. Atualmente trabalha no Instituto Nacional de Controle de Qualidade em Saúde (INCQS) da Fundação Oswaldo Cruz.

\section{Rafaela Pinto da Costa}

Mestre do Programa de Vigilância Sanitária do Instituto Nacional de Controle de Qualidade em Saúde (INCQS) da Fundação Oswaldo Cruz. Graduada em Medicina Veterinária pela Universidade Federal Fluminense. Atualmente trabalha na Fundação Ataulpho de Paiva.

\section{Tatiana Avila da Cruz}

Graduada em Engenharia de Alimentos pela Universidade Estácio de Sá.

\section{Marcus Henrique Campino de la Cruz}

Doutor do Programa de Química do Instituto de Química da Universidade Federal do Rio de Janeiro. Graduado em Química com Atribuições Tecnológicas pela Universidade Federal do Rio de Janeiro. Atualmente trabalha no Instituto Nacional de Controle de Qualidade em Saúde (INCQS) da Fundação Oswaldo Cruz.

\section{Luiz Antônio d'Ávila}

Doutor do Programa de Química do Instituto de Química da Universidade Federal do Rio de Janeiro. Graduado em Química pela Universidade Federal do Rio de Janeiro. Atualmente trabalha na Escola de Química da UFRJ. 


\section{Resumo}

A norma NBR ISO/IEC 17025:2005 especifica os requisitos gerenciais e técnicos para a implementação do sistema de gestão da qualidade em laboratórios de ensaio e calibração e tem como um dos requisitos a garantia da qualidade dos resultados. Materiais de referência (MR) e materiais de referência certificados (MRC) são recomendados para essa finalidade. A utilização de MR e MRC vem aumentando nos laboratórios para o controle de qualidade, calibração de equipamentos e desenvolvimento de metodologias, atividades que demandam medidas confiáveis e rastreáveis. A produção desses materiais na área de resíduos de medicamentos veterinários é muito inferior à demanda. No Brasil ainda não há produtores de $\mathrm{MR}$ de resíduos de medicamentos veterinários em alimentos, dificultando o acesso a esses produtos e aumentando o custo para os laboratórios nacionais. O objetivo desse trabalho foi produzir um MR do antibiótico oxitetraciclina na matriz leite, usando um leite naturalmente contaminado e utilizando como técnica de preservação a liofilização. A determinação da concentração foi realizada pela técnica de cromatografia a líquido acoplada a espectrometria de massas sequencial (CLAE-EM/EM). Os lotes de MR 1 e 2 produzidos apresentaram-se suficientemente homogêneos, com concentração média de 177.3 e $165.1 \mu \mathrm{g} / \mathrm{L}$, respectivamente. Os lotes foram considerados suficientemente estáveis durante seis meses armazenados a $(-20 \pm$ $\left.1^{\circ} \mathrm{C}\right)$ e $\left(6 \pm 1^{\circ} \mathrm{C}\right)$ e durante sete dias a $\left(6 \pm 1^{\circ} \mathrm{C}\right)$ e $\left(40 \pm 1^{\circ} \mathrm{C}\right)$. Conclui-se que a técnica de liofilização se mostrou adequada como processo de secagem para produção de MR contendo oxitetraciclina em matriz leite. A produção deste MR de oxitetraciclina em leite no Brasil permitirá a melhoria da qualidade das análises dos laboratórios de alimentos e consequentemente no suporte às ações de controle e fiscalização sanitária, além da possibilidade da elaboração de um ensaio de proficiência.

Palavras-chave: Material de referência. Oxitetraciclina. Leite. 


\section{Abstract}

The NBR ISO/IEC 17025:2005 standard describes the managerial and technical prerequisites for implementing quality management systems in test and calibration laboratories and stresses the importance of assuring the quality of the analytical results. Reference materials $(\mathrm{RM})$ and certified reference materials (CRM) are recommended for this purpose. RM and CRM are being used increasingly in laboratories for analytical quality control, equipment calibration and methodology development, all of which require reliable, traceable measurements. Demand for these materials for veterinary drug residues far outstrips current production capacity. In Brazil there are not yet any producers of RM for veterinary drug residues in food, which hampers access to such products and raises costs for the country's laboratories. The aim of this study was to produce a RM for an antibiotic, oxytetracycline, in a milk matrix, using a naturally contaminated milk and lyophilization (freeze-drying) as the preservation technique. The determination of the concentration was performed by liquid chromatography coupled with sequential mass spectrometry (LC-MS/MS). The lots of MR 1 and 2 produced were sufficiently homogeneous, with an average concentration of 177.3 and $165.1 \mu \mathrm{g} / \mathrm{L}$, respectively. The batches were considered sufficiently stable for six months stored at ($\left.20 \pm 1^{\circ} \mathrm{C}\right)$ and $\left(6 \pm 1^{\circ} \mathrm{C}\right)$ and for seven days at $\left(6 \pm 1^{\circ} \mathrm{C}\right)$ and $\left(40 \pm 1^{\circ} \mathrm{C}\right)$. It was concluded that the lyophilization technique proved to be adequate as a drying process for the production of oxytetracycline containing milk matrix. The production of this MR of oxytetracycline in milk in Brazil will allow the improvement of the quality of the analyzes of the food laboratories and consequently in the support to the actions of control and sanitary inspection, besides the possibility of the elaboration of a proficiency test.

Keywords: Reference material. Oxytetracycline. Milk. 


\section{Introdução}

A utilização de antibióticos à base de tetraciclinas (TC) na medicina veterinária vem se tornando crescente, para fins terapêuticos e profiláticos em animais produtores de alimentos, sendo incluídas nos principais programas de monitoramento de resíduos (SPISSO et al, 2007; FRITZ:ZUO, 2007; SPISSO et al, 2009). Assim, para proteger a saúde humana dos efeitos adversos de antimicrobianos, agências reguladoras de cada país têm estabelecido Limites Máximos de Resíduos (LMR) para as substâncias autorizadas para o uso como medicamentos veterinários em animais produtores de alimentos (D'ANGELINO:NUNES, 2007). O Brasil utiliza os LMR estabelecidos pelo Codex Alimentarius (CODEX ALIMENTARIUS, 2017) e pela Resolução 54/00 do GMC/Mercosul (MERCOSUL, 2001) internalizada pela Instrução Normativa MAPA n 12/01 (BRASIL, 2001).

Os LMR das tetraciclinas em leite fixados pelo Codex Alimentarius, Mercosul e Ministério de Agricultura, Pecuária e Abastecimento (Mapa) do Brasil (BRASIL, 2001) são de $100 \mu \mathrm{g} / \mathrm{L}$, para as substâncias tetraciclina, clortetraciclina e oxitetraciclina separadamente ou o somatório delas. A União Europeia adota um LMR para as tetraciclinas de $100 \mu \mathrm{g} / \mathrm{L}$, considerando como resíduo marcador a soma da substância original (oxitetraciclina, tetraciclina e clortetraciclina) e seu 4-epímero, embora o valor numérico adotado seja o mesmo. Já o Food and Drug Administration dos Estados Unidos (FOOD AND DRUG ADMINISTRATION, 2017) adota um LMR para as tetraciclinas de $300 \mu \mathrm{g} / \mathrm{L}$, sendo para as substâncias tetraciclina, clortetraciclina e oxitetraciclina separadamente ou o somatório delas.

No Brasil, existem dois programas nacionas de monitoramento de resíduos de medicamentos veterinários em alimentos, o Plano Nacional de Controle de Resíduos em Produtos de Origem Animal (PNCR) do Mapa (BRASIL, 1995) e o Programa de Análise de Resíduos de Medicamentos Veterinários em Alimentos de Origem Animal (PAMVet) da Agência Nacional de Vigilância Sanitária (Anvisa) (BRASIL, 2003). O monitoramento de resíduos de tetraciclinas em leite é um elemento chave para assegurar a proteção da saúde dos consumidores. É necessária a utilização de métodos apropriados com ênfase na seletividade e sensibilidade para identificar com confiança e quantificar compostos de tetracicilinas em uma matriz complexa como o leite (SPISSO et al, 2009; ZELENY et al, 2006). 
Nos últimos anos, as análises de resíduos de tetraciclinas em leite vêm sendo utilizadas tanto com técnicas de triagem, por exemplo os testes de Enzyme-Linked Immunosorbent Assay (Elisa) e biossensores, e pricipalmente quanto a cromatografia à líquido acoplada à espectrometria de massas sequencial (SPISSO et al, 2009).

Os Materiais de Referência (MR)/Materiais de Referência Certificados (MRC) são uma das principais ferramentas para a garantia e controle de qualidade descritas na norma ABNT NBR ISO/IEC 17025 (ABNT, 2005), onde a sua utilização está destinada a: calibração, validação de metodologias, rastreabilidade, demonstração de proficiência de laboratórios e fornecimento de valores a outros materiais.

A demanda no Brasil por MR na área de resíduos em alimentos excede a oferta em termos da variedade de materiais e disponibilidade, diferentemente da Europa que possui uma grande oferta de MRC nesta área. Apesar do grande empenho do Instituto Nacional de Metrologia, Normalização e Qualidade Industrial (Inmetro), que é o responsável no país em prover confiança à sociedade brasileira nas medições e nos produtos, através da metrologia e da avaliação da conformidade, promovendo a harmonização das relações de consumo, a inovação e a competitividade do País (INMETRO, 2017), somente um MRC de alimentos no país na área de resíduos de agrotóxicos em tomate foi produzido (CARDOSO, 2008).

Uma das grandes dificuldades dos laboratórios analíticos brasileiros é a indisponibilidade desses MRC no mercado nacional. Além dos preços elevados destes materiais importados, há ainda problemas no desembaraço alfandegário (CARDOSO, 2008).

O leite foi a matriz selecionada para a produção do candidato a MR por ser o alimento mais consumido entre as crianças e os idosos e por ser uma das matrizes analisadas nos dois principais programas nacionais de controle de resíduos de medicamentos veterinários em alimentos, o PNCR do Mapa e o PAMVet da Anvisa. O antibiótico selecionado para a produção foi a oxitetraciclina, por ser a substância mais identificada desta classe pelo Laboratório de Resíduos de Medicamentos Veterinários em Alimentos do Instituto Nacional de Controle de Qualidade em Saúde (INCQS) (SPISSO et al, 2007; SPISSO et al, 2009) e por ter o maior número de produtos registrados de acordo com o Sindicato Nacional das Indústrias de Produtos para a Saúde Animal (SINDAN, 2017). 


\section{Metodologia}

\section{PREPARO DO CANDIDATO A MR}

$\mathrm{O}$ medicamento tetraciclina $\mathrm{LA} / \mathrm{SPV}^{\circledR}$ (medicamento à base de oxitetraciclina injetável, de ação prolongada pronta para uso) foi dosado no Laboratório de Medicamentos do INCQS seguindo os procedimentos da The United States Pharmacopeia Convention (USP, 2016). A análise foi realizada utilizando a técnica por cromatografia a líquido por detecção por ultravioleta e a concentração de oxitetraciclina obtida foi de $1,87 \pm 0,2 \mathrm{~g} / \mathrm{L}$.

Antes da administração do medicamento no animal foi realizada a análise de uma amostra proveniente de uma ordenha do animal por CLAE-EM/EM (SPISSO et al, 2009) para comprovar a isenção de antibióticos no leite. O medicamento foi administrado via intramuscular no animal de espécie bovina e de raça mestiço de holandês zebu com peso médio de $500 \mathrm{~kg}$ no curral do Departamento de Zootecnia da Universidade Federal Rural do Rio de Janeiro (UFRRJ), sendo que após $1 \mathrm{~h}$ da aplicação foi realizada a ordenha (5L). O leite obtido foi centrifugado a $4500 \mathrm{x} g$ por 5 min e a $4^{\circ} \mathrm{C}$ para remoção da gordura e armazenado em um freezer de ultra-baixa temperatura $a<-70^{\circ} \mathrm{C}$ (ColdLab, Brasil).

O leite foi analisado em duplicata de injeção pelo método de determinação de resíduos de tetraciclinas em leite por Cromatografia à Líquido de Alta Eficiência acoplada à Espectrometria de Massas Sequencial (SPISSO et al, 2009).

\section{ENVASE, CONGELAMENTO E LIOFILIZAÇÃO DO CANDIDATO A MATERIAL DE REREFÊNCIA}

Volumes de $2 \mathrm{~mL}$ do leite desnatado foram pesados em frascos de vidro âmbar de capacidade de $5 \mathrm{~mL}$, totalizando 116 frascos por lote dispostos em quatrobandejas. Em seguida, os frascos foram congelados em freezer de ultra-baixa temperatura a < $70^{\circ} \mathrm{C}$ (ColdLab, Brasil). Posteriormente, os frascos foram liofilizados por $24 \mathrm{~h}$ (Liotop, Brasil), sob as seguintes condições: temperatura $<-40^{\circ} \mathrm{C}$ e pressão inferior a $100 \mu \mathrm{Hg}$. Ao completar o período do ciclo de liofilização os frascos foram fechados a vácuo, através da utilização de sistema de vedação manual acoplado ao equipamento. Os frascos 
foram novamente pesados para verificar a perda de água durante o processo e lacrados com uma tampa metálica.

\section{VERIFICAÇÃO DO VÁCUO}

Após a retirada dos frascos do liofilizador foi realizada a verificação de vácuo aplicando uma centelha elétrica em cada um, com auxílio de uma bobina de Tesla (Coil, 2-12-8, Brasil). Os frascos que apresentaram vácuo foram lacrados com tampa de metal, etiquetados, e estocados em freezer de ultra-baixa temperatura a $<-70^{\circ} \mathrm{C}$ (ColdLab, Brasil). Os frascos que não atingiram o vácuo foram descartados.

\section{HOMOGENEIDADE}

No planejamento do estudo de homogeneidade foi idealizado o preparo de dois lotes dos candidatos a MR. Para a avaliação da homogeneidade dos lotes preparados, 16 frascos de cada lote foram selecionados aleatoriamente utilizando a ferramenta do Excel ${ }^{\circledR}$, sendo quatro frascos de cada bandeja para obtenção de uma análise representativa. $\mathrm{O}$ leite foi reconstituído com aproximadamente a mesma quantidade de massa de água purificada tipo I que foi sublimada durante o processo de liofilização, homogeneizado com agitação mecânica e analisado em duplicata conforme a metodologia previamente descrita (SPISSO et al, 2009).

A avaliação estatística no estudo de homogeneidade dos resultados das análises de oxitetraciclina foi realizada de acordo com os critérios da ABNT ISO GUIA 35 (ABNT, 2012).

O procedimento para a determinação do grau de homogeneidade abordado na ABNT ISO GUI 35 (ABNT, 2012) foi realizada a análise de variância (Anova) fator único, utilizando o programa Microsoft $\operatorname{Excel}^{\circledR}$, as amostras foram consideradas suficientemente homogêneas, se o $F_{\text {calculado }}<F_{\text {crítico, }}(\alpha=5 \%)$, evidenciando que não há diferenças significativas entre os lotes preparados (LAPPONI, 2000). Nesse cálculo também é demonstrado o valor de $p$ que é a probabilidade do $F_{\text {crítico }}>F_{\text {tabelado, }}$ e para que isso ocorra o valor de $p$ tem que ser maior do que o nível de significância observado, sendo geralmente adotado 0,05 . 


\section{ESTABILIDADE}

\section{Curta Duração}

O estudo de estabilidade de curta duração que simula as condições de transporte foi realizado em duas temperaturas distintas: $6 \pm 1^{\circ} \mathrm{C}$ e $40 \pm 1^{\circ} \mathrm{C}$, em um período de sete dias. Para a simulação do transporte em diferentes temperaturas, foi utilizada a metodologia do "isochronous design" ou modelo isócrono (LAMBERTY et al, 1998). A cada dia dois frascos que estavam estocados a $6^{\circ} \mathrm{C}$ e $40^{\circ} \mathrm{C}$ foram transferidos para temperatura de $\leq 70^{\circ} \mathrm{C}$ (temperatura de referência). Após os sete dias de armazenamento, os frascos foram analisados ao mesmo tempo, sob as mesmas condições de análise.

\section{Longa Duração}

O estudo de estabilidade de longa duração foi realizado por um período de seis meses em duas temperaturas distintas: $-20 \pm 1^{\circ} \mathrm{C}$ e $6 \pm 1^{\circ} \mathrm{C}$, utilizando a abordagem clássica (determinações do analito ao longo do tempo) e a abordagem isócrona (medições são realizadas sob condições de repetitividade, em uma mesma análise) (LAMBERTY et al, 1998).

As avaliações estatísticas dos estudos de estabilidade de curta duração e de longa duração dos candidatos a MR foram realizadas de acordo com a ABNT ISO GUIA 35 (ABNT, 2012), através da análise de variância (Anova) dos resíduos da regressão linear. Neste caso, estuda-se a variação de estabilidade dos analitos ao longo do tempo. Não ocorrendo variação da concentração do analito em função do tempo, o material é considerado estável.

\section{Resultados e discussão}

A análise do leite ordenhado antes da aplicação do medicamento no animal gerou um cromatograma sem a presença do pico cromatográfico da oxitetraciclina 
(OTC) e também apresentou resultado abaixo do limite de detecção $(3,7 \mu \mathrm{g} / \mathrm{L})$, concluindo-se à ausência da substância no leite analisado.

No leite ordenhado após a administração do medicamento, foi possível visualizar um pico de OTC (Figura 1), podendo confirmar a presença da substância no leite. A concentração obtida de OTC foi de 186,0 $\mu \mathrm{g} / \mathrm{L}$ no leite ordenhado.

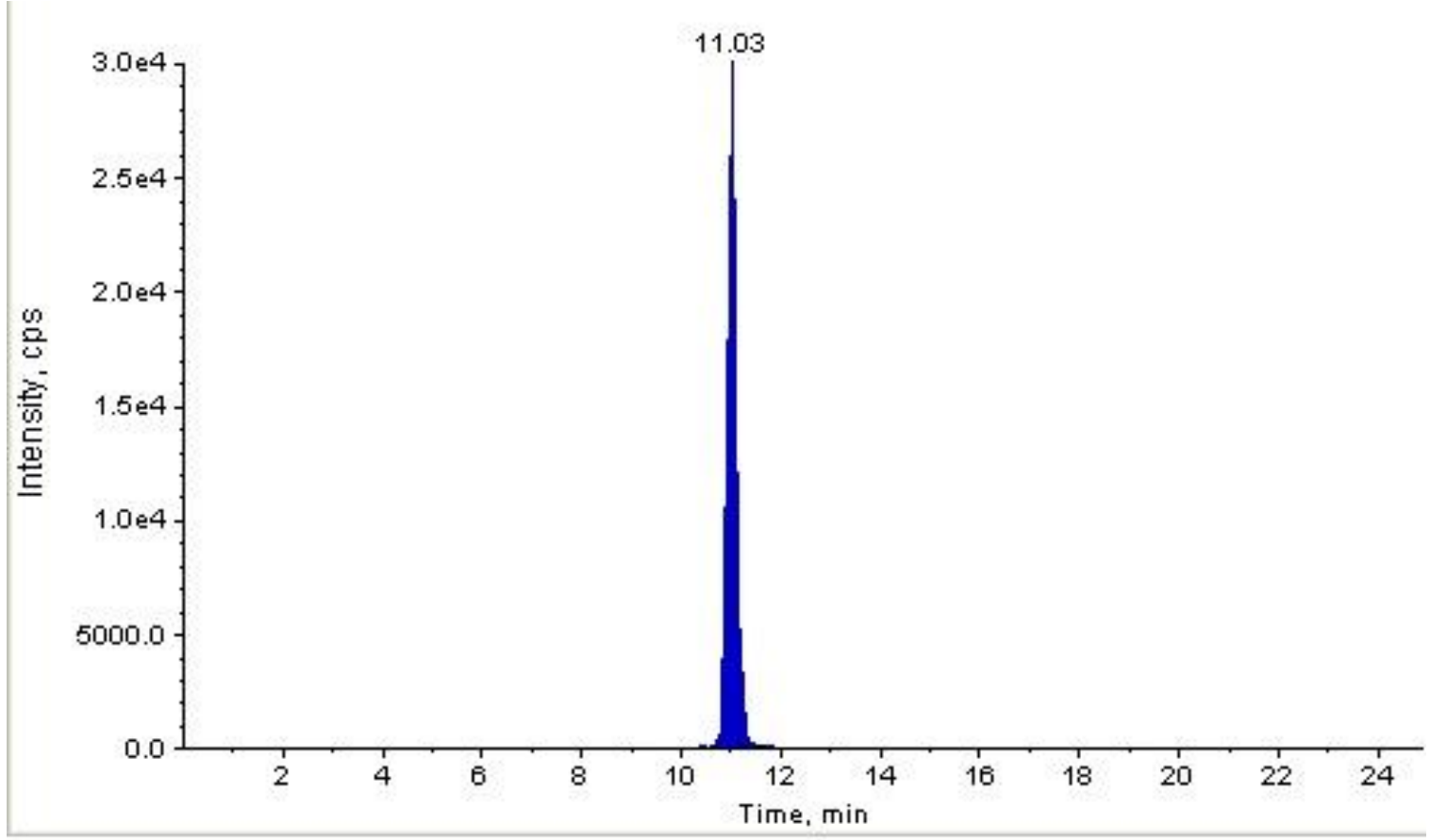

Figura 1. Cromatograma da Oxitetraciclina no leite após a administração do medicamento

No lote 1 todos os frascos apresentaram presença de vácuo $(100,0 \%)$ e no lote 2, em três frascos não foi detectada presença de vácuo (97,4\%). Estes resultados indicaram que o processo de liofilização do eficaz para preservação do material. Outros autores utilizaram a liofilização como processo de secagem e preservação de MR em matriz leite contendo bactérias e também obtiveram resultados satisfatórios, com presença de vácuo variando de 92,3-100,0\% dos frascos dos lotes produzidos (ROSAS et al., 2010; BRANDAO et al., 2013a,b; BRANDAO et al., 2014).

A Tabela 1 demonstrou os resultados de concentração de OTC para o estudo de homogeneidade dos lotes do MR preparados. O coeficiente de variação $(\mathrm{CV})$ máximo obtido em cada lote foi de $16,7 \%$. Os lotes 1 e 2 foram considerados suficientemente homogêneos pela ABNT ISO Guia 35 (ABNT, 2012), pois o valor de $\mathrm{F}_{\text {calculado }}$ foi menor do que o $\mathrm{F}_{\text {crítico, }}$ e o valor de $p$ maior do que 0,05 ; como demonstrado na Tabela 2. 
MONTEIRO, M. A. et al. Produção do material de referência de oxitetraciclina em leite em pó desnatado. R. Científica UBMBarra Mansa (RJ), ano XXII, v. 19, n. 36, 1. Sem. 2017. p. 146-160.

ISSN 1516-4071

Tabela 1 - Estudo de Homogeneidade

\begin{tabular}{|c|c|c|c|c|c|c|c|c|c|c|c|}
\hline \multicolumn{6}{|c|}{ Lote 1} & \multicolumn{6}{|c|}{ Lote 2} \\
\hline $\begin{array}{l}\text { MR (n.o } \\
\text { item de } \\
\text { ensaio) }\end{array}$ & $\begin{array}{r}\text { Conc } \\
(1\end{array}$ & $\begin{array}{l}\text { tração } \\
\text { /L) }\end{array}$ & $\begin{array}{l}\text { Média } \\
(\mu \mathrm{g} / \mathrm{L})\end{array}$ & $\begin{array}{l}\text { Desvio } \\
\text { padrão } \\
(\mu \mathrm{g} / \mathrm{L})\end{array}$ & $\begin{array}{l}\text { CV } \\
(\%)\end{array}$ & MR & $\begin{array}{r}\text { Conce } \\
\quad(\mu\end{array}$ & $\begin{array}{l}\text { ração } \\
\text { L) }\end{array}$ & $\begin{array}{l}\text { Média } \\
(\mu \mathrm{g} / \mathrm{L})\end{array}$ & $\begin{array}{l}\text { Desvio } \\
\text { padrão } \\
(\mu \mathrm{g} / \mathrm{L})\end{array}$ & $\begin{array}{l}\text { CV } \\
\text { (\%) }\end{array}$ \\
\hline MR3 & 188,0 & 189,1 & 188,6 & 0,7 & 0,4 & MR233 & 173,1 & 165,1 & 169,1 & 5,7 & 3,3 \\
\hline MR11 & 214,5 & 197,4 & 206,0 & 12,1 & 5,9 & MR236 & 140,4 & 143,4 & 141,9 & 2,1 & 1,5 \\
\hline MR18 & 157,0 & 159,0 & 158,0 & 1,4 & 0,9 & MR243 & 171,6 & 175,7 & 173,6 & 2,8 & 1,6 \\
\hline MR27 & 170,2 & 165,1 & 167,6 & 3,6 & 2,1 & MR248 & 95,1 & 91,1 & 93,1 & 2,8 & 3,0 \\
\hline MR31 & 179,3 & 169,2 & 174,3 & 7,1 & 4,1 & MR249 & 186,7 & 173,5 & 180,1 & 9,3 & 5,2 \\
\hline MR47 & 160,8 & 180,8 & 170,8 & 14,1 & 8,3 & MR266 & 193,9 & 171,9 & 182,9 & 15,5 & 8,5 \\
\hline MR48 & 166,6 & 147,5 & 157,1 & 13,5 & 8,6 & MR275 & 175,6 & 171,6 & 173,6 & 2,8 & 1,6 \\
\hline MR50 & 162,1 & 187,1 & 174,6 & 17,7 & 10,1 & MR284 & 151,0 & 169,0 & 160,0 & 12,7 & 8,0 \\
\hline MR64 & 179,3 & 167,3 & 173,3 & 8,5 & 4,9 & MR287 & 155,9 & 152,9 & 154,4 & 2,1 & 1,4 \\
\hline MR66 & 153,7 & 194,9 & 174,3 & 29,1 & 16,7 & MR289 & 168,9 & 170,9 & 169,9 & 1,4 & 0,8 \\
\hline MR69 & 163,6 & 175,6 & 169,6 & 8,5 & 5,0 & MR299 & 175,1 & 166,1 & 170,6 & 6,4 & 3,7 \\
\hline MR77 & 197,7 & 166,8 & 182,3 & 21,9 & 12,0 & MR301 & 193,3 & 152,5 & 172,9 & 28,9 & 16,7 \\
\hline MR90 & 186,7 & 208,1 & 197,4 & 15,1 & 7,7 & MR322 & 176,2 & 201,2 & 188,7 & 17,7 & 9,4 \\
\hline MR105 & 176,1 & 196,1 & 186,1 & 14,2 & 7,6 & MR333 & 182,2 & 176,9 & 179,5 & 3,8 & 2,1 \\
\hline MR109 & 190,0 & 173,0 & 181,5 & 12,0 & 6,6 & MR341 & 167,3 & 167,3 & 167,3 & 0,0 & 0,0 \\
\hline MR113 & 177,3 & 172,3 & 174,8 & 3,5 & 2,0 & MR344 & 174,6 & 152,7 & 163,6 & 15,5 & 9,5 \\
\hline
\end{tabular}

Tabela 2 - Análise de Variância dos resultados do estudo de homogeneidade dos lotes 1 e 2

\begin{tabular}{ccccccc}
\hline Fonte da variação & SQ & gl & MQ & $F$ & valor-P & $F_{\text {crítico }}$ \\
Entre lotes & 421.9 & 1 & 421.9 & 2.77 & 0.11 & 4.18 \\
Dentro dos lotes & 4420.3 & 29 & 152.4 & & & \\
Total & 4842.2 & 30 & & & & \\
\hline $\begin{array}{c}\text { Legenda: } S Q \\
\text { gl }\end{array}$ & $=$ soma quadrática de liberdade & & & & & \\
MQ $=$ média quadrática & & & & & &
\end{tabular}

A Figura 2 mostra os gráficos dos estudos de estabilidade para a oxitetraciclina, nos períodos de curta e de longa duração. A Tabela 3 apresenta as condições e os resultados do estudo de estabilidade. No estudo de estabilidade de curta duração, o material nas temperaturas de $6^{\circ} \mathrm{C}$ e $40^{\circ} \mathrm{C}$ foi considerado suficientemente estável durante o período de estudo (sete dias), pois o intervalo de confiança a $95 \%$ abrange o 
MONTEIRO, M. A. et al. Produção do material de referência de oxitetraciclina em leite em pó desnatado. R. Científica UBMBarra Mansa (RJ), ano XXII, v. 19, n. 36, 1. Sem. 2017. p. 146-160.

ISSN 1516-4071

valor zero. Os estudos de estabilidade de longa duração clássica e isócrona nas temperaturas $-20^{\circ} \mathrm{C}$ e $6^{\circ} \mathrm{C}$ demonstraram que os materiais foram considerados suficientemente estáveis. No modelo clássico, o maior valor em módulo do coeficiente angular obtido foi na temperatura de $-20^{\circ} \mathrm{C}$, comprovando assim, uma variação maior da concentração ao longo do tempo. Já no modelo isócrono, os valores de coeficientes angulares obtidos nas temperaturas $-20^{\circ} \mathrm{C}$ e $6^{\circ} \mathrm{C}$ foram praticamente iguais, evidenciando a obtenção de menores variações nesse modelo de estabilidade.

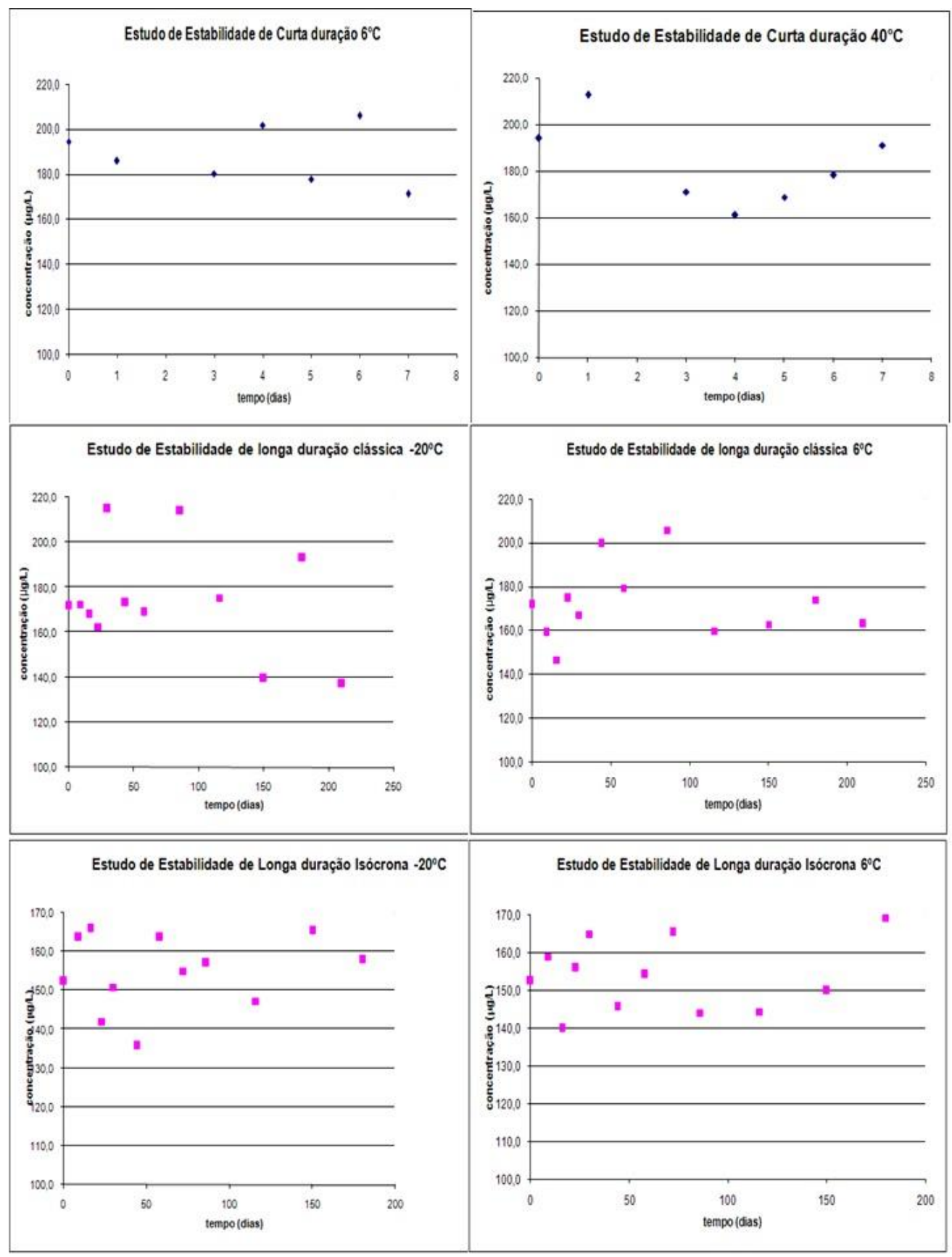

Figura 2. Gráfico dos estudos de estabilidade de oxitetraciclina 
Tabela 3 - Resultado do Estudo de Estabilidade

\begin{tabular}{ccccccc}
\hline Estudo de estabilidade & \multicolumn{2}{c}{ Curta Duração } & \multicolumn{3}{c}{ Longa Duração } \\
\hline Modelo & \multicolumn{2}{c}{ Isócrona } & \multicolumn{2}{c}{ Clássica } & \multicolumn{2}{c}{ Isócrona } \\
\hline Temperatura & $6 \circ \mathrm{C}$ & $40 \circ \mathrm{C}$ & $-20 \circ \mathrm{C}$ & $6 \circ \mathrm{C}$ & $-20 \circ \mathrm{C}$ & $6 \circ \mathrm{C}$ \\
Coeficiente Angular & $-0,92$ & $-3,08$ & $-0,10$ & 0,0000038 & 0,040 & 0,033 \\
Limite Inferior (95\%) & $-6,62$ & $-10,26$ & $-0,33$ & $-0,169$ & $-0,088$ & $-0,095$ \\
Limite Inferior (95\%) & 4,77 & 4,10 & 0,127 & 0,169 & 0,17 & 0,16 \\
Resultado & estável & estável & estável & estável & estável & estável \\
\hline
\end{tabular}

\section{Conclusão}

A técnica de liofilização se mostrou adequada como processo de secagem para produção de MR contendo oxitetraciclina em matriz leite suficientemente homogênea. O material poderá ser transportado na temperatura de até $40^{\circ} \mathrm{C}$, por um período máximo de sete dias aos laboratórios que venham a adquirir estes MR. O material poderá ser armazenado tanto a $-20^{\circ} \mathrm{C}$ quanto a $6^{\circ} \mathrm{C}$, por um período de seis meses. Um estudo por um tempo prolongado é necessário para avaliar até quanto tempo este material se mantém estável nestas condições. Os baixos valores de coeficiente angular obtidos em ambas as temperaturas indicam que os MR deverão ser manter estáveis nestas condições de armazenamento por muito mais tempo.

A produção deste MR de oxitetraciclina em leite no Brasil permitirá a melhoria da qualidade das análises dos laboratórios de alimentos e consequentemente no suporte às ações de controle e fiscalização sanitária, além da possibilidade da elaboração de um ensaio de proficiência.

\section{Agradecimentos}

À professora Dra Verônica Lobato e aos profissionais do Departamento de Zootecnia da Universidade Federal Rural do Rio de Janeiro (UFRRJ). 


\section{Referências}

\section{ASSOCIAÇÃO BRASILEIRA DE NORMAS TÉCNICAS. ISO/IEC 17025:}

Requisitos gerais para a competência de laboratórios de ensaio e calibração. Rio de Janeiro, 2005.

Associação Brasileira de Normas Técnicas. ISO GUIA 35: Materiais de ReferênciaPrincípios Gerais e Estatísticos para Certificação. Rio de Janeiro, 2012.

BRANDAO MLL; ROSAS CO; BRICIO SML; COSTA JCB; MEDEIROS VM; WARNKEN MB. Produção de materiais de referência para avaliação de métodos microbiológicos em alimentos: estafilococos coagulase positiva e Listeria spp. em leite em pó. Revista Analytica 2013a; 63(Fevereiro/Março):60-71.

BRANDAO, MLL; ROSAS, CO; BRICIO, SML; COSTA, JCB; MEDEIROS, VM; WARNKEN, MB; DE LA CRUZ, MHC; NOBREGA, AW. Avaliação de Crioprotetores na Produção de Material de Referência para Enumeração de Coliformes em Leite em Pó a ser Utilizado em Ensaio de Proficiência. Rev Inst Adolfo Lutz, v. 72, n. 2, p.144-50, 2013b.

BRANDAO, MLL; ROSAS, CO; BRICIO, SML; COSTA, JCB; MEDEIROS, VM; DE LA CRUZ, MHC; NOBREGA, AW. Produção de material de referência para ensaio de proficiência para enumeração de Bacillus cereus em leite em pó. Vig Sanit Debate, v. 2, n. 1, p. 39-45, 2014.

BRASIL. Ministério da Agricultura, Pecuária e Abastecimento. Instrução Normativa no 12, 1 abril de 2001. Adota o Regulamento Técnico Mercosul Metodologias Analíticas, Ingestão Diária Admissível e Limites Máximos de Resíduos de Medicamentos Veterinários em Alimentos de Origem Animal. Diário Oficial da União, 12 abril 2001, seção 1 .

BRASIL. Portaria Ministerial n. 527, de 15 de agosto de 1995, do Ministério da Agricultura, Pecuária e Abastecimento do Brasil (MAA).

BRASIL. Resolução RDC n. 253, de 16 de setembro de 2003. Dispõe sobre a criação do Programa de Análise de Resíduos de Medicamentos Veterinários em Alimentos de Origem Animal - PAMVet. 
CARDOSO, MHWM. Preparação de um Material de Referência Certificado para Controle de Agrotóxicos em Hortifrutigranjeiros. 2008. 191 p. Tese (doutorado em vigilância sanitária). Instituto Nacional de Controle de Qualidade em Saúde FIOCRUZ, Rio de Janeiro.

CODEX ALIMENTARIUS. Limite Máximo de Resíduos em Drogas Veterinárias. Disponível em:

<http://www.accessdata.fda.gov/scripts/cdrh/cfdocs/cfcfr/CFRSearch.cfm>. Acesso em: 21 mai. 2017.

D'ANGELINO, JL; NUNES, MT. Ocorrência de resíduos de antibióticos no leite, em fazendas produtoras e no leite pronto para consumo. Hig. Alim., v. 21, n. 149, p. 57-61, 2007.

FOOD DRUGS AND ADMINISTRATION. Limite Máximo de Resíduos Substâncias Farmacologicamente Ativas em Alimentos. Título 21, Capítulo 1, Seção 556. Disponível em:

<http://www.accessdata.fda.gov/scripts/cdrh/cfdocs/cfcfr/CFRSearch.cfm>. Acesso em: 21 mai. 2017.

FRITZ, JH; ZUO, Y. Simultaneous determination of tetracycline, oxytetracycline, and 4-epitetracycline in milk by high-performance liquid chromatography. Food Chemistry, v. 105, p. 1297-1301, 2007.

INSTITUTO NACIONAL DE METROLOGIA, NORMALIZAÇÃO E QUALIDADE INDUSTRIAL - Materiais de Referência Certificado. Disponível em: <http://www.inmetro.gov.br/metcientifica/mrc.asp>. Acesso em: 21 mai. 2017.

LAMBERTY, A; SCHIMMEL, H; PAUWELS, J. The study of the stability of reference materials by isochronous measurements. Fresenius J. Anal. Chem., v. 360, p. 359-361, 1998.

LAPPONI, JC. Estatística usando Excel. São Paulo. Lapponi Treinamento e Editora, 2000.

MERCOSUL. Resolução n54/2000. Aprova o Regulamento Técnico Mercosul sobre Metodologias Analíticas, Ingestão Diária Admissível e Limites Máximos de Resíduos 
para Medicamentos Veterinários em Alimentos de Origem Animal. Brasília, 29 setembro 2000.

ROSAS, CO, BRANDAO, MLL, BRICIO, SML, MEDEIROS, VM, BERNARDO, SPC, DE LA CRUZ, MHC et al. Desenvolvimento de material de referência para ensaio de proficiência em microbiologia de alimentos. Rev Inst Adolfo Lutz. 2010;69(1):15-22.

SINDICATO NACIONAL DA INDÚSTRIA DE PRODUTOS PARA SAÚDE ANIMAL. Compêndio de Produtos Veterinários. Disponível em: <http://www.cpvs.com.br/cpvs/> Acesso em: 21 mai. 2017.

SPISSO, B F; JESUS, ALO; ARAUJO JUNIOR, MAG; MONTEIRO, MA. Validation of a high-performance liquid chromatographic method with fluorescence detection for the simultaneous determination of tetracyclines residues in bovine milk. Anal. Chim. Acta, v. 581, p.108-117, 2007.

SPISSO, BF; ARAUJO JUNIOR, MAG; MONTEIRO, MA; LIMA, AMB; PEREIRA, UM; LUIZ, RA; NOBREGA, AW. A liquid chromatography-tandem mass spectrometry confirmatory assay for the simultaneous determination of several tetracyclines in milk considering keto-enol tautomerism and epimerization phenomena. Anal. Chim. Acta, v. 656, p.72-84, 2009.

ZELENY, R, ULBERTH, F, GOWIK, P, POLZER, J, VAN GINKEL, LA, EMONS, H. Developing new materials for effective veterinary drug-residue testing in food-producing animals. Trends Anal. Chem., v. 25, n. 9, p. 927-36, 2006.

THE UNITED States Pharmacopeia 32. National Formulary 34: 2016. Rockville:U.S. Pharmacopeia, 2016. 2v. 